\title{
The Mesenteric-Caval Fistula: First Results of a New Technique in a Transperitoneal Reconstruction of the Caval Vein by Fulminant Thrombosis of the Inferior Vena Cava Based on Homozygous Antithrombin III-Deficiency
}

\author{
Justus Gross, Rainer Petzina, Rouven Berndt, Bernd Panholzer, Andreas Bayer, \\ Katharina Huenges, Leonie Aschauer, Jochen Cremer, Rene Rusch
}

Department of Cardiac and Thoracic Vascular Surgery, UKSH, Kiel, Germany

Email: Justus.gross@uksh.de

Received 1 July 2016; accepted 9 August 2016; published 12 August 2016

Copyright (C) 2016 by authors and Scientific Research Publishing Inc.

This work is licensed under the Creative Commons Attribution International License (CC BY). http://creativecommons.org/licenses/by/4.0/

(c) (i) 0 pen Access

\begin{abstract}
Recurrent thrombotic occlusions are one major problem in patients with thrombosis of the inferior vena cava. Due to this, we report a new surgical strategy for the construction of aorto-caval (mesenteric-caval) fistula in a patient with homozygous Antithrombin III (ATIII)-Deficiency. The patient survived postoperatively and only surgical complications grade I and II (Clavien-Dindo classification) were reported after short-term and one year follow-up. After one year, the CT-angiography did not show any caval thrombosis or stenosis and no restriction or occlusion of the fistula. Thus, the mesenteric-caval fistula could be safely performed and resulted in a satisfactory patency.
\end{abstract}

\section{Keywords}

Mesenteric-Caval Fistula, Thrombosis, Transperitoneal Reconstruction, Homozygous Antithrombin III-Deficiency

\section{Introduction}

Fulminant thrombosis of the inferior caval vein is a rare but serious condition and often correlated with threatHow to cite this paper: Gross, J., Petzina, R., Berndt, R., Panholzer, B., Bayer, A., Huenges, K., Aschauer, L., Cremer, J. and Rusch, R. (2016) The Mesenteric-Caval Fistula: First Results of a New Technique in a Transperitoneal Reconstruction of the Caval Vein by Fulminant Thrombosis of the Inferior Vena Cava Based on Homozygous Antithrombin III-Deficiency. Surgical Science, 7, 342-347. http://dx.doi.org/10.4236/ss.2016.78049 
ening complications [1]. In particular, thrombotic caval occlusions are correlated with a high embolism risk. Besides primary thrombectomy, the construction of arterio-venous (AV)-fistula is essential for preventing rethrombosis and improving patency rate during long-time course. However, long-time data of these patients have not been available until today. Despite performing AV-fistula, clinical practice has shown that a number of patients suffer from thrombotic re-occlusions of the caval vein.

Congenital homozygote ATIII-Deficiency is a rare, but clinically very relevant cause of thrombophilia $(0.1 \%$ of total population, approx. $1 \%$ in thrombotic collective) [2]. Circa $80 \%$ of patients suffer at least one venal thromboembolism after the age of 40 [3] [4]. Here, we present the individual treatment of a young female patient with ATIII-Deficiency and fulminant thrombosis of the inferior vena cava undergoing a new surgical technique performing a thrombectomy and construction of a mesenteric-caval fistula.

Based on the former experience with thrombosis of the inferior vena cava [5], we have optimized the concept of AV-fistula. The aim of this report was to present our refined technique and evaluate the perioperative outcome and patency rate during long time follow-up.

\section{Patient and Indication}

A 24-year-old female patient with homozygous AT-III-deficiency was admitted to our Casualty department in November 2014. A pronounced medical history has been established. In 2009 a transitory ischemic attack and a miscarriage occurred, which were caused by a thrombotic complication. Throughout the course, the patient received a dual antiplatelet therapy (DAPT) with $100 \mathrm{mg}$ ADA and $75 \mathrm{mg}$ Plavix each $1 \times 1 / \mathrm{d}$ as well as therapeutically-administered Clexane. In 2010 the patient, again pregnant, suffered a localized thrombotic cerebral infarction and was treated by mechanical thrombectomy then by stent inclusion in the middle cerebral artery. During the in-patient stay an intrauterine fetal death occurred, patho-histologic analysis showed a placental infarction. Starting in 2012 due to a categorical pregnancy wish, she received the anticoagulant warfarin by injection. Two years later the pregnancy was completed with cesarean delivery of a healthy child.

Only a few weeks post partum the patient visited the emergency room with flank pain and dyspnea. Initial sonography showed a wall-adhered thrombus in the inferior vena cava as well as a kidney infarction on the right superior pole. Due to the described dyspnea symptoms, a computer tomography of the lungs was done and a pulmonary embolism (PE) in the right inferior lobe with right infarction pneumonia was found hence she was transferred to the division of cardiovascular diseases. A transthoracic EKG could rule out right heart strain. Furthermore good left-ventricle global function without wall-movement disruption was shown. The inferior vena cava was not congested and breathing modulated. Additionally a transesophageal EKG could rule out intra-cardial thrombosis. Due to the previous pulmonary embolisms the until-then blood-thinning therapy was changed to $15 \mathrm{mg}$ Xarelto $2 \times 1 / \mathrm{d}$.

A few days later the patient was readmitted again with progressive left flank pain. A new computer tomography (CT) showed a progressive pulmonary embolism in the left inferior lobe, as well as a pronounced congestive edema around both kidneys with fully thrombosed renal veins up to the inferior vena cava, of which its lumen were almost fully obstructed (Figure 1(a) and Figure 1(b)). After consulting with our clinic, operative therapy was initiated. Postoperative Complications were evaluated with the Clavien-Dindo Classification of Surgical Complications. One year after surgery a new CT-angiography was performed for long-time follow-up.

According to the ethical guidelines of our institutional ethical committee, both patients had signed the informed consent for use of their data.

\section{Surgical Technique}

On the same day as transfer the urgent operation was done. After problem-free laparotomy, the pelvic veins, inferior vena cava and the aorta including the illiac artieries were exposed using the Kocher manoeuver. The left renal vein acted as an anatomical variant in the common iliac vein is, the V. ovarica dextra was varicose and completely thombosed (Figure 2(a) and Figure 2(b)).

After sequential clamping off of the inferior vena cava, cava thrombectomy as well as thrombecotomy of the right renal vein was done. The V. ovarica dextra was tied up close to the basis and removed. A brief retrograde flushing from the thoracic inferior vena cava was supported by Trendelenburg positioning and high-peep ventilation. To reconstruct the inferior vena cava, bovine pericardial patches were used. The renal veins were detached at the junction to the common iliac vein, the thrombic segment resected close to the hilus and the remaining 


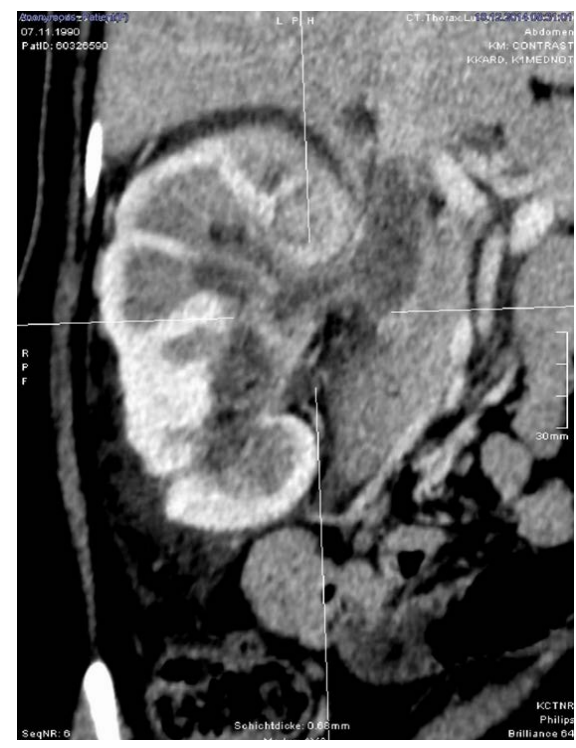

(a)

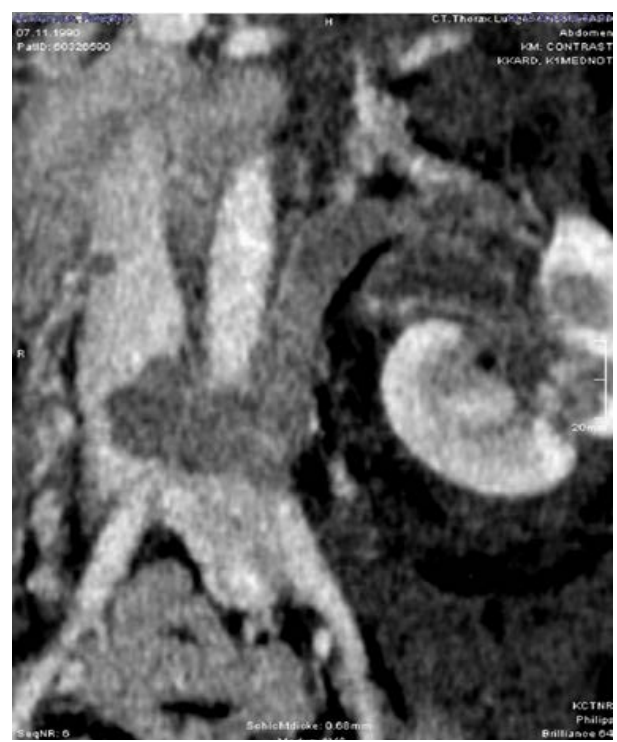

(b)

Figure 1. Thrombosis of right renal vein extending to the inferior vena cava (a) and (b).

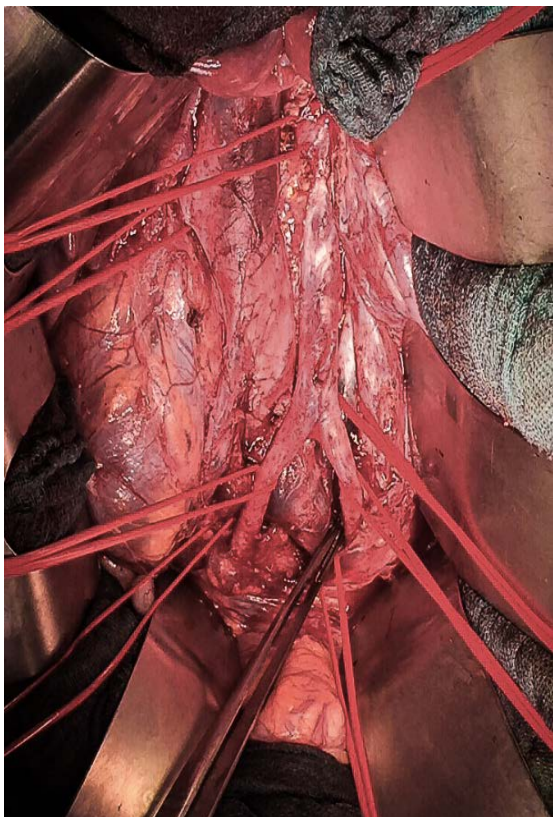

(a)

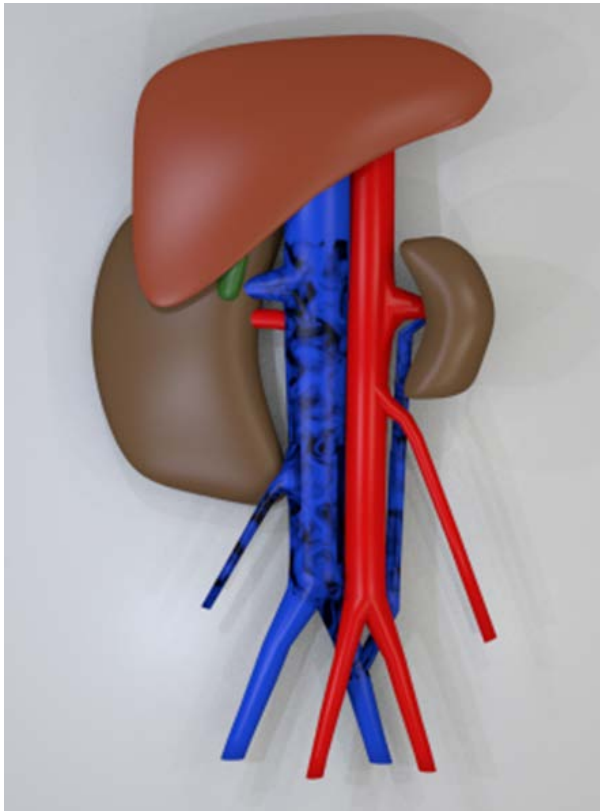

(b)

Figure 2. Intra-operative site (a) and as sketch (b).

vessel-stump embolectomized. The reconstruction followed with an $8 \mathrm{~mm}$ Dacron-interposition (end-to-end on the left renal vein stump and end-to-side on the inferior vena cava patch). To construct an AV fistula, the inferior mesenteric artery was detached after clinical verification of sufficient Riolan's arch and also inserted into the cava-patch (end-to-side) (Figure 3(a) and Figure 3(b)). During the operation therapeutic heparin was administered with 1000IE ATIII, blood re-transfusion was ensured by a cell-saver-system.

\section{Clinical Assessment}

After a lengthy but technically uncomplicated operation, the patient was stable and was transferred to the cardiac and thoracic vascular surgery intensive care unit under moderate catecholamine administration. Through 


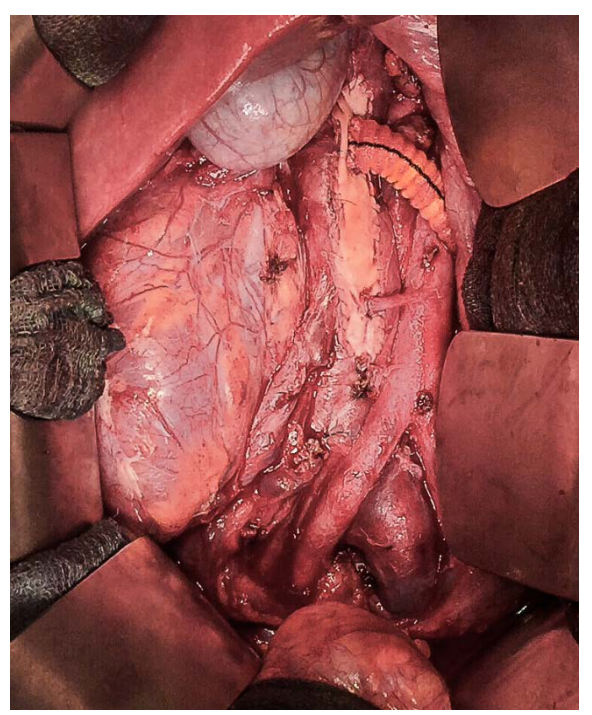

(a)

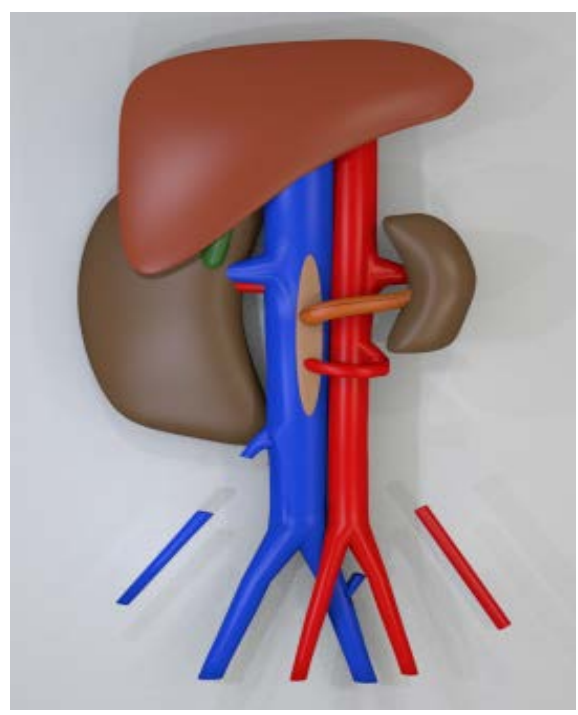

(b)

Figure 3. Condition after construction of the inferior vena cava patch-plastics, reinsertion of the Dacron bypass of the left renal vein and construction of a mesentericocaval fistula between the A. mesenterica inf. and inferior vena cava (patch-inserted), intra-operative site (a) and as sketch (b).

stabilization of renal retention parameters, a progressive kidney replacement could be avoided. The echocardiograph progress control showed a continued right-heart strain. On the morning of the third post-operative day, Milrinon therapy could be gradually reduced and ended. Generally there was compensated biventricular pump function without indications of valve insufficiency or pericardial effusion. The patient received two red cell concentrates during the stay in the intensive care unit stay. After consultation with colleagues in the hematology department, dual therapy with $100 \mathrm{mg}$ ADA and 75 mg Plavix was supplemented by therapeutic Cumarin-derived anticoagulant Warfarin. The regular in-patient ultrasound duplex abdominal checks showed stenose-free reconstructions in the operated area as well as no further indications of thrombosis formation near the inferior vena cava or the renal veins. Thus, only Grade I and II complications (Clavien-Dindo classification) were reported. On the 14th postoperative day the young woman was released from the hospital with good wound healing and reported no physical discomfort.

After one year a new CT-angiography was performed. All anastomosis were free of stenosis and no thrombotic occlusion of the caval vein was detected, moreover the mesenterico-caval fistula was open (Figure 4). The patient did not present any symptoms during the one-year follow up. No complications were reported under the anticoagulation.

\section{Discussion}

The patient's congenital homozygote antithrombin deficiency is indeed a rare, but clinically very relevant cause of thrombophilia (prevalence $0.1 \%$ total population, ca. $1 \%$ in thrombotic collective). The serine protease inhibitor (serpin) inactivates the factor Xa and other coagulant factors through generation of a 1:1 complex mainly thrombin. Complex formation is strongly fostered by therapeutically administered heparin (conformation of the reactive antithrombin) and explains the heparin effect mechanism.

With congenital (heterozygote) antithrombin deficiency, venal thromboembolisms occur primarily between 15 and 30 years-of-age. A dysfunctional antithrombin molecule that actually interferes with the heparin docking site (type $2 \mathrm{~b}$ ), implies a tendentially lesser thromboembolism risk (relevant risk 5 - 10 times higher) than a molecule with a defective thrombin docking site or combined docking defect (type 2a or 2c, increase of relevant risk ca. 20 times higher). On the other hand, the homozygote antithrombin deficiency is generally precludes survival. There are very few publications about the manifestations and long-term survival regarding imperative thrombosis risk [3] [4] [6]. 


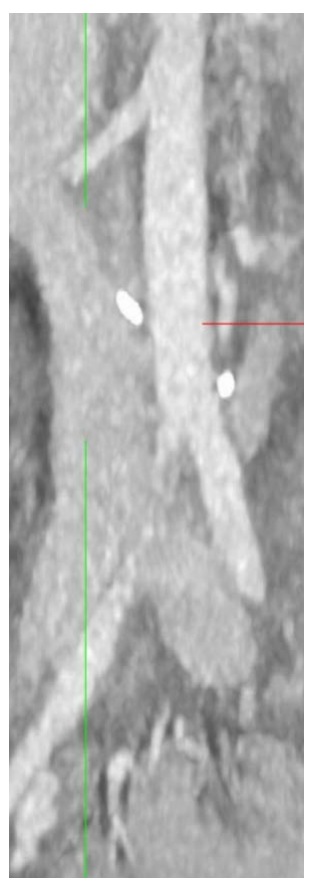

Figure 4. CT-scan control after 12 months shows open fistula and the inferior vena cava free of thrombosis (RAO and CC-multi slice reconstruction).

With drug therapy the anticoagulants Marcumar and warfarin should be mentioned. The only difference between the two compounds is a significantly longer half-life of the Phenprocoumon (150 hours) versus warfarin (37 to 50 hours). Hence, Wafarin therapy is easier to control [7].

Medicinal treatment of the young patient was complicated by firstly, a questionable cognitive medicinal compliance and secondly, by the desire to become pregnant. Warfarin is contraindicated due to its teratogenicity (multiple deformities with exposure after the $8^{\text {th }}$ week of pregnancy), the critical phase for warfarin embryopathy is assumed to be the $6^{\text {th }}$ to $12^{\text {th }}$ week after conception [8]. In view of an accompanying stent implantation in the middle cerebral artery, DAPT and low molecular heparin (Clexane) were used. After completion of the pregnancy and clinically apparent PE, therapy with Xarelto was initiated, under which a progressive thrombosis of renal veins and the inferior vena cava could not be prevented.

There are different therapy approaches for actual renal thrombosis. The main goal of the therapy is preservation of the renal parenchyma and function. Due to the fact that there are few and mostly retrospective studies additionally with small case numbers or individual case studies, no generally applicable therapy recommendations can extrapolated. Generally over the past 25 years the trend has been from a primarily operative to a rather conservative medicinal therapeutic approach [9]. Surgical treatment includes thrombectomy and the usually-associated nephrectomy. Indications for nephrectomy are life-threatening hemorrhages from renal capsule rupture or complications such as hypertension and renal infection that cannot be overcome conservatively. Thrombectomy should only be considered for obstructions that come off the main veins since it is not operatively possible to evacuate thrombosis of the small arcuate veins and the interlobular veins. The goal should be initial conservative stabilization followed by facilitation of commonly-observed generation of collaterals [10].

Thromboses in the inferior vena cava are rare, yet they are particularly dangerous due to a high embolism risk. Transabdominal and/or transfemoral thrombectomy (with AV fistula) is safe, indicated and occasionally lifesaving. Patency of the inferior vena cava can in most cases be achieved. Usually the operation is carried out under general anasthesia with positive end-expiratory pressure and systemic heparinization. The venous thrombectomy procedure is well known. After open desobliteration (endovenectomy) of the common femoral vein allowing free flow in the deep thigh-veins, the shallow veins are tied up. A large arteriovenous fistula (at least 3 $4 \mathrm{~mm}$ in diameter) should prevent re-thrombosis. The main branch of the great saphenous vein, an important collateral path, must be protected. There were reports of rare cases of a deep vein thrombosis in combination with a free-floating caval thrombus ascending up to the renal veins. In these cases arteriovenous fistulas could 
be placed on both groin blood vessels [11].

A case overview from Kniemeyer et al. presents a review of 70 patients with thrombosis of the inferior vena cava, treated over 15 years by transperitoneal caval thrombectomy and/or transfemoral venal thrombectomy. In 64 patients a transfemoral thrombectomy with AV fistula construction was planned, additional transabdominal access followed in 41 cases. 3 patients died perioperatively (4.3\%). An intraoperative pulmonary embolism was assumed for 3 patients, one of which died. A cava opening rate of $87.1 \%$ could be achieved postoperatively. Later results were satisfactory. 58 patients (82.9\%) could be observed over time (average observation time $44 \pm$ 35 or 3 - 120 months). 3 patients died during observation. For 47 patients ( 69 extremities, $>1$ year postoperative) $17.4 \%$ of their extremities gave no discomfort, $40.6 \%$ had a slight PTS, $30.4 \%$ moderate and $11.6 \%$ severe PTS (Ulcus ruris in $7.2 \%$ ). The long-term opening rate was good, peripheral venous congestion complaints were not rare [11] [12].

Because of the complexity of abdominal venous thrombosis, we intra-operatively decided to construct a mesentericocaval fistula over the A. mesenterica inferior after the sufficiency Riolan's arch could be clinically checked (spraying arterial return flow after stopping of the same). The postoperative openness of the embolectomized inferior vena cava and the renal veins as well as the postoperative right-heart strain in echocardiogram supports the feasibility of our mesenterico-caval fistula construction. Comparable case examples have rarely been published. There are no long-term results on the efficacy of this procedure.

The individual treatment of the female patient with ATIII-Deficiency shows that complex open surgical thrombectomy and reconstruction of the renal veins and the Vena cava as well as construction of a mesentericocaval fistula over the inferior mesenteric artery for a very rare but severe thrombophilia condition has led to success in the sense of a curative organ-preserving measure. The long-term openness under the combined therapy with DAPT and Warfarin remains to be seen.

\section{Conclusion}

Congenital homozygotic antithrombin deficiency is a very rare, yet clinically very relevant cause of thrombophilia. Rare homozygotic AT-III-deficiency requires an explicitly heme statistical solution, and we chose a combination of lifelong Warfrin and single antiplatelet therapy. The open, operative and time-consuming inferior vena cava and renal-vein embolectomy with construction of a mesentericocaval fistula using the inferior mesenteric artery proved to be successful. We consider the significance of the open surgical inferior vena cava and renal vein embolectomy as a definitively curative procedure.

\section{References}

[1] Chow, F.C., Chan, Y.C., Cheung, G.C. and Cheng, S.W. (2015) Mid- and Long-Term Outcome of Patients with Permanent Inferior Vena Cava Filters: A Single Center Review. Annals of Vascular Surgery, 29, 985-994. http://dx.doi.org/10.1016/j.avsg.2015.01.009

[2] Hirsh, J., Piovella, F. and Pini, M. (1989) Congenital Antithrombin III Deficiency. Incidence and Clinical Features. The American Journal of Medicine, 87, 34S-38S. http://dx.doi.org/10.1016/0002-9343(89)80529-7

[3] Barthels, M., et al. (2003) Das Gerinnungskompendium, Thieme Verlag, Stuttgart, 529 ff.

[4] Lindhoff, E., et al. (2008) Update Thrombophilie. Hämostaseologie, 28, 365-375.

[5] Alcocer, F., Perez, S. and Martinez, C. (2012) Small Skin Incision and Fistula Elevation for Hemodialysis Using the Femoral Vein. Journal of Vascular Surgery, 56, 753-756. http://dx.doi.org/10.1016/j.jvs.2012.01.077

[6] Bruhn, D.B. and Schambeck, C.M. (2007) Hämostaseologie für die Praxis: Sicher durch den klinischen Alltag Gebundene Ausgabe. Schattauer-Verlag, Stuttgart, 425-427.

[7] Wehling (2005) Klinische Pharmakologie. Thieme Verlag, Stuttgart, S. 107.

[8] Wehling (2005) Klinische Pharmakologie. Thieme Verlag, Stuttgart, S. 660.

[9] Witz, M., et al. (1996) Renal Vein Occlusion: A Review. The Journal of Urology, 155, 1173-1179. http://dx.doi.org/10.1016/S0022-5347(01)66206-2

[10] Keating, M.A. and Althausen, A.F. (1985) The Clinical Spectrum of Renal Vein Thrombosis. 6, 938-945.

[11] Kniemeyer, H.W., et al. (1993) Thrombectomy with Arteriovenous Fistula for Embolizing Deep Venous Thrombosis: An Alternative Therapy for Prevention of Recurrent Pulmonary Embolism. The Clinical Investigator, 72, 40-45.

[12] Kniemeyer, H.W., Abbara, M., Luther, B., Torsello, G., Grabitz, K. and Sandmann, W. (1997) Chirurgische Behandlung der V.-cava-inferior-Thrombose. Gefässchirurgie, 2, 69-77. 


\section{Submit or recommend next manuscript to SCIRP and we will provide best service for you:}

Accepting pre-submission inquiries through Email, Facebook, LinkedIn, Twitter, etc.

A wide selection of journals (inclusive of 9 subjects, more than 200 journals)

Providing 24-hour high-quality service

User-friendly online submission system

Fair and swift peer-review system

Efficient typesetting and proofreading procedure

Display of the result of downloads and visits, as well as the number of cited articles

Maximum dissemination of your research work

Submit your manuscript at: http://papersubmission.scirp.org/ 